\title{
HISTORIAS DE VIDA EN EDUCACIÓN MUSICAL. ANA LUCÍA FREGA: FORMACIÓN E INFLUENCIAS
}

\author{
Life stories in music education. \\ Ana Lucia Frega: formation and influences
}

\section{Susana Sarfson Gleizer*}

Fecha de recepción: 28/09/2017 • Fecha de aceptación: 13/02/2018

Resumen. Este artículo se enmarca dentro de la investigación en el ámbito de la historia de la educación musical, y tiene el propósito de rescatar algunas memorias de la Dra. Ana Lucía Frega (Buenos Aires, 1935), pedagoga musical con varias décadas de trayectoria profesional, cuya influencia en la didáctica de la música se extiende internacionalmente. La perspectiva que se emplea en este artículo es cualitativa, con elementos de investigación etnográfica; los datos se recogen mediante entrevistas, y se triangula la información con otras fuentes (bibliográficas, hemerográficas, entrevistas breves a otras personas). Se busca indagar en el punto de partida de su desarrollo profesional internacional y comprender cómo su contacto con insignes maestros (Maurice Martenot, Oliver Messiaen, Arnold Bentley) pudo influir en su perspectiva personal en el ejercicio de la profesión docente y como formadora de profesorado de música e investigadores. Su presencia en la International Society of Music Education (ISME), de la cual fue Presidente, potenció su influencia en ámbitos gubernamentales, tanto en su propio país como en otros. La Historia de la Educación se construye, entre otros elementos, con las contribuciones de maestros y maestras reconocidos, y en este sentido este trabajo busca comprender el propio punto de vista de una de ellas, plasmado en sus recuerdos y reflexiones personales acerca de su propia formación musical y sus primeras etapas profesionales, en las que la interacción social fue importante para la consolidación de sus logros.

Palabras clave: Educación musical; Historia de la educación; Música; Investigación musical.

\footnotetext{
* Departamento de Expresión Musical, Plástica y Corporal, Facultad de Ciencias Humanas y de la Educación de Huesca, Universidad de Zaragoza. Calle Valentín Carderera, 4. 22003 Huesca. España. sarfson@unizar.es
}

Cómo citar este artículo: Sarfson Gleizer, Susana. «Historias de vida en educación musical. Ana Lucía Frega: formación e influencias». Historia y Memoria de la Educación 8 (2018): 683-698. 
Abstract. This article fits within the framework of music education history research, and aims at rescuing certain memories of Dr. Ana Lucía Frega (Buenos Aires, 1935), a music pedagogue whose professional career spanned several decades. The article uses a qualitative approach, with elements of ethnographic research, while the data and information collected through interviews are contrasted with other sources. The paper analyses the starting point of her international professional development and attempts to determine how her contact with renowned masters (Maurice Martenot, Oliver Messiaen, Arnold Bentley) influenced her personal perspective in the teaching profession and as a teacher of music teachers and researchers. Her presence in the International Society of Music Education (ISME), of which she was President, strengthened her influence in governmental areas. The history of education is built, among other elements, with the contributions of recognized teachers. In this sense, this work seeks to understand the point of view of one of these docents, embodied in her memories and personal reflections about her own musical formation and her early professional stages. Social interaction proved to be a key factor in the consolidation of hers and others' achievements.

Keywords: Music Education; History of Education; Music; Music Research.

\section{INTRODUCCIÓN}

La educación musical tiene que ver con la comunicación, y en este sentido entendemos que en todas las épocas ha habido buenos maestros y maestras (con mayor o menor reconocimiento público), que han tenido una profunda influencia en sus discípulos y discípulas, tanto por sus cualidades musicales como pedagógicas. ${ }^{1}$ Pero en un ámbito eminentemente heterogéneo como es la formación y las experiencias del profesorado de música, es interesante conocer el recorrido personal para comprender mejor las producciones de cada docente. En especial, este artículo se centra en la figura de la profesora Ana Lucía Frega (Buenos Aires, 1935), y en las experiencias que pudieron favorecer o impulsar su devenir profesional. Se trata de una figura que ha influido (e influye) en la enseñanza de la música no sólo en su propio país, sino en el contexto internacional (fundamentalmente en países Iberoamericanos, España y Estados Unidos) por su proyección a través de la ISME (International Society of Music Education), a la cual

\footnotetext{
${ }^{1}$ Susana Sarfson Gleizer, Educación Musical en Aragón (1900-1950). Legislación, publicaciones y escuela (Zaragoza: Prensas Universitarias, 2010).
} 
pertenece desde la década de 1960, y de la cual fue electa Presidente (1994-2000). Si bien la mayor fuente de información, en este caso, es autobiográfica (diálogos, recuerdos), se han contrastado los datos objetivos y se procura dar un énfasis a ciertos aspectos interpretativos de los mismos. El objetivo es conocer un punto de vista, que habría que integrar o entrelazar con otros relatos o historias de vida, para comprender un período en que la educación musical tuvo un impulso notable en Argentina, en particular en su capital, Buenos Aires, donde diversas corrientes musicales y pedagógicas enriquecieron el panorama de la época referida.

En este trabajo se utiliza una metodología predominantemente cualitativa, con perspectiva etnográfica, es decir, se busca describir el contexto vital de la persona, teniendo en cuenta que el ambiente influye en el sujeto y en sus grupos de pertenencia, y esto se realiza con la finalidad de poder interpretar y comprender algunos procesos. Hay que tener en cuenta que en una historia de vida, la persona realiza un relato sobre el desarrollo de su biografía desde su propio punto de vista. ${ }^{2}$ Pero la historia de vida es portadora de la subjetividad de quien narra, y de su propia cosmovisión: por esto se trata de contrastar esta información con otras fuentes. Sin embargo, posibilita la comprensión de procesos de individuación: cómo se construye la profesionalización, en este caso particular, de la docencia. Implica contextualización en términos ideológicos, políticos y culturales. ${ }^{3}$ También se tienen en cuenta los rasgos de un enfoque etnográfico, en tanto que «las tradiciones, roles, valores y normas del ambiente en que se vive se van internalizando poco a poco y generan regularidades que pueden explicar la conducta individual y de grupo en forma adecuada». ${ }^{4}$

Asimismo, se tiene en cuenta que el conocimiento sociológico no tiene una única perspectiva, y que es importante el cruce de la persona como

\footnotetext{
${ }^{2}$ José Ignacio Ruíz Olabuénaga, «Historias de vida», en Metodología de la Investigación Cualitativa, ed. José Ignacio Ruíz (Bilbao: Universidad de Deusto, 2012): 306. 207.

${ }^{3}$ Francisco Cisterna Cabrera, «Categorización y triangulación como procesos de validación del conocimiento en investigación cualitativa», Theoria 14, no. 1 (2005): 61-71.

${ }^{4}$ Miguel Martínez Miguélez, «El método etnográfico de investigación», http://investigacionypostgrado. uneg.edu.ve/intranetcgip/documentos/225000/225000archivo00002.pdf (consultado el 5 de mayo de 2017).
} 
individuo con las estructuras sociales. ${ }^{5} \mathrm{Al}$ registrar recuerdos de una persona, y recogerlos en forma oral, se trata de documentar esas memorias, así como las reflexiones, de acuerdo con su propia mirada. ${ }^{6}$ Además, es importante considerar las experiencias y contextos del pasado, para enriquecer y revalorizar las prácticas docentes de la práctica cotidiana. ${ }^{7}$

El objetivo principal es mostrar un testimonio que, aunque tenga carácter subjetivo, refleja sucesos, pero también significados. Como se ha dicho, se procura triangular la información en aquellos aspectos que es viable hacerlo, y aportar elementos para la comprensión del período en el que se han desarrollado los acontecimientos evocados. El contraste de información se realiza fundamentalmente sobre las cuestiones referidas por la entrevistada, por lo que las luces dominan sobre las posibles sombras.

Para obtener la información biográfica se realizó una serie de entrevistas, diálogos fluidos con la Dra. Ana Lucía Frega, desarrolladas a lo largo de varios meses durante el año 2017. Las entrevistas fueron semiestructuradas, a partir de algunas preguntas para centrar la conversación en las primeras experiencias musicales, la formación inicial, las primeras experiencias profesionales, pero al mismo tiempo han sido entrevistas con un fluir espontáneo. La información mencionada se ha ido ampliando, cuando ha sido necesario, a través de otras fuentes documentales: fuentes hemerográficas, entrevistas breves con condiscípulas, bibliografía.

\section{EVOCACIÓN DE LA INFANCIA}

Ana Lucía Frega nació en la ciudad de Buenos Aires el 25 de noviembre de 1935. Llegaba a una familia que ya tenía dos hijos: dos chicos que en ese momento tenían 9 y 14 años. Transcribimos sus palabras acerca de los primeros recuerdos:

\footnotetext{
${ }^{5}$ Eliane Veras, «Historia de vida: ¿un método para las ciencias sociales?», Cinta Moebio 39 (2010): 142-152.

${ }^{6}$ Stephen Zdzinski, «Joseph A. Labuta and His Life in Music Education: An Oral History», Journal of Historical Research in Music Education XXIX, no. 2 (2008): 108-128.

${ }^{7}$ María Eugenia Bolaño Amigo, «Estética ilustrada, discursos de clase y educación musical escolar en los comienzos del siglo xx», Historia y Memoria de la Educación 5 (2017): 385-400.
} 
Desde chica escuché a mi mamá cantar, hablar de poesía y de música. Yo a los 3 años bailaba y cantaba. Me encantaba bailar todo. Desde una tarantela hasta "En un mercado persa», con vestidos de gitana. Y sentaba a todas las muñecas en el patio y les daba clase. Fui al jardín de infantes desde los 3 años. Tenía dos hermanos mayores con gran diferencia de edad: cuando yo tenía 3, mis hermanos tenían 12 y 17 años. Mi papá dijo: «Esta nena con estos dos hermanos varones tiene que ir al colegio para conocer nenas».

Llama la atención en su contexto el haber acudido al Jardín de Infantes ${ }^{8}$ desde tan temprana edad: se trata de un nivel educativo que no era obligatorio, y que muchas veces se obviaba, o se realizaba solamente a los 5 años, es decir, uno antes de comenzar la escolaridad obligatoria. Evidentemente se sustancia la intención de la familia de ampliar las experiencias vitales y formativas de la niña.

$\mathrm{Al}$ indagar acerca de sus comienzos en el piano, la entrevistada responde:

Tocaba primero en un pianito de juguete (a los 4 años). En esa época mi tío tocaba, tenía una pianola-piano, y tocaba mucho, yo escuchaba y jugaba. Después tuve un piano La Primera, fabricado en Santa Fe.

Este testimonio remite a la demanda de pianos que hubo en esa época en Argentina y a un ejemplo singular dentro de la industria del país, vinculada a las características del tejido social del momento, cuya información merece la pena ampliar debido a su singularidad.

La fábrica de pianos La Primera estaba ubicada en la localidad de Pilar (provincia de Santa Fe) que es una de las colonias fundadas por Guillermo Lehmann (1840-1886). Esta fábrica fue fundada en 1939: en el pueblo habían cerrado unos talleres de ferrocarril que daban trabajo a la mayor parte de la población, y un grupo de sus ciudadanos más notables pensaron que una fábrica de pianos podía dar trabajo, y además cubrir

\footnotetext{
${ }^{8}$ Nivel educativo no obligatorio, actualmente en España se denomina Educación Infantil, y en Argentina, Nivel Inicial.
} 
la necesidad creciente de pianos de la Argentina, en una época en que la clase media que prosperaba en este país consideraba que la práctica musical en casa era una parte importante de la vida de las familias. Así tomaron contacto con quien sería su director técnico, José María Alcayde, que construía pianos en la ciudad de Santa Fe desde 1937 en su propio taller, con una calidad reconocida. La fábrica de Pilar, La Primera, terminó su primer piano en 1940, llegó a tener 200 empleados y a fabricar 1000 pianos por año. Tras varias décadas de florecimiento, los cambios sociales fueron llevando a la disminución de demanda y a su decadencia económica.

\section{PRIMERA FORMACIÓN MUSICAL SISTEMÁTICA}

Ana Lucía Frega estudió en la Escuela de Música del diario La Pren$s a,{ }^{9}$ en el bello edificio inaugurado en 1898 en la Avenida de Mayo, en una zona emblemática de la ciudad de Buenos Aires. Su profesora de piano fue María Rosa Farcy de Montal, quien dirigía este centro de estudios desde $1924 .^{10}$

La profesora Frega recuerda: «En ese mismo conservatorio estudió María Teresa Criscuolo. En solfeo estudiamos el Lemoine, y el Lavignac manuscrito a ocho claves. Llegué a cantar solfeo acompañándome al piano».

Es notable destacar que su primera profesora de piano, María Rosa Farcy de Montal, fue autora de varias publicaciones didácticas para el aprendizaje del piano y de la entonación musical, editadas por Ricordi: ${ }^{11}$

\footnotetext{
${ }^{9}$ Periódico diario, fundado en Buenos Aires en 1869.

${ }^{10}$ El diario La Prensa era uno de los más importantes del país en ese momento, ofrecía una serie de servicios para sus lectores: Consultorio Médico-quirúrgico (dirigido por el doctor Julio Darnet), un Consultorio de Química industrial (dirigido por el doctor Carlos E. Pinto), un Consultorio Agrícola-ganadero (dirigido por el doctor Roberto Ezcurra), un Consultorio Jurídico, dos Salones de actos públicos (uno en el segundo piso, para asambleas populares no muy numerosas, reuniones de comisiones y conferencias; y el gran salón de fiestas, situado en el primer piso, para veladas o conciertos), una Escuela gratuita de música (dirigida por María Rosa Farcy de Montal desde 1908), el Instituto Popular de Conferencias (fundado el 18 de julio de 1914) y la Biblioteca (Saítta, 1996). Es relevante mencionar que actualmente el edificio del diario La Prensa es la Casa de la Cultura del Gobierno de la ciudad de Buenos Aires, declarado Monumento Histórico Nacional en 1995, y que el gran salón de actos o Salón Dorado conserva todavía uno de los pianos de cola de aquel conservatorio.

${ }^{11}$ La editorial Ricordi Americana comenzó como sucursal de Ricordi de Milán en 1924.
} 
es decir, la calidad pedagógica de su magisterio fue reconocida a tal punto de ser objeto de ediciones por una de las editoriales musicales más prestigiosas y con mayor difusión.

\section{SU EXPERIENCIA EN EL CONSERVATORIO NACIONAL DE MÚSICA E INICIOS PROFESIONALES}

Después de esta formación inicial en la Escuela de Música del diario La Prensa, Ana Lucía Frega continuó sus estudios en el Conservatorio Nacional de Música y Arte Escénico «Carlos López Buchardo», donde obtuvo el título de Profesora Superior de Piano en 1954.

El director del Conservatorio en ese momento era el maestro Juan Francisco Giacobbe (1907-1990). ${ }^{12}$ Entre los profesores notables que evoca la Dra. Frega, el maestro José Antonio Torre Bertucci merece una mención especial en sus recuerdos. Fue compositor, profesor de Acústica, Lenguaje Musical y Contrapunto, formador de una generación de músicos argentinos, pero también un exquisito coleccionista de arte oriental: con su colección personal de obras de arte, que donó para ser expuesta y estudiada, se constituye una gran cantidad de los fondos del Museo de Arte Oriental, y una parte del Museo de Arte Decorativo. ${ }^{13}$

La profesora Frega relata:

Con José Torre Bertucci cursé Acústica y Lenguaje Musical IV. Era admirable, de él aprendí mucha didáctica. Nos hacía cantar, mientras él tocaba el piano, y explicaba el lenguaje y los recursos expresivos de cada una de las obras que interpretábamos. Nunca era una clase puramente teórica, siempre había música práctica y se cuidaba la calidad expresiva de cada interpretación.

La biografía del compositor José Antonio Torre Bertucci, como la de otros insignes músicos argentinos (compositores, intérpretes, profesores) queda aún por ser ordenada y estudiada en profundidad; sin embargo, es

\footnotetext{
${ }^{12}$ Compositor, director, filósofo, considerado un hombre del Renacimiento, había sido alumno (y amigo) de Maurice Ravel. Queda pendiente un estudio profundo acerca de su biografía, su obra, y sus aportaciones a la cultura.

${ }^{13} \mathrm{Ver}$ https://www.cultura.gob.ar/quien-fue-torre-bertucci-el-coleccionista-que-dono-casi-2000-piezas-al-museo-de-arte-oriental_3219/.
} 
importante mencionar que Torre Bertucci había sido alumno del insigne compositor Alberto Williams (1862-1952), es autor de un importante catálogo de obras compositivas entre las que destacan sus Sonatas para piano, y también de un célebre Tratado de Contrapunto, obra de referencia para los estudios de composición, editado por Ricordi.

El contexto familiar de Ana Lucía Frega fue determinante en los inicios laborales, mientras continuaba sus estudios en el Conservatorio Nacional de Música.

Mi papá se murió cuando yo tenía 15 años. El profesor Jurafsky, ${ }^{14}$ que más tarde fue director del Conservatorio, me mandó un telegrama, y recuerdo cómo me saludó cuando llegué al conservatorio. Lo mismo mi maestro de piano, Jorge Fanelli. Mi mamá tardó dos años en cobrar la pensión. Así que me puse a trabajar, dando algunas clases particulares, en esa época había muchísimo trabajo. Me propuse se la mejor maestra de música posible, porque me gustaba.

En cuanto finalizó sus estudios en el Conservatorio Nacional de Música «Carlos López Buchardo», Ana Lucía Frega comenzó su labor profesional como maestra de música en escuelas municipales de la ciudad de Buenos Aires (en los niveles educativos de Jardín de Infantes y Primaria), así como en la Escuela Argentina Modelo, ${ }^{15}$ labor que realizó durante muchos años. También ejerció como profesora de música en diversos colegios públicos y privados de nivel secundario. Esto le fue dando una experiencia en contextos educativos y sociales diversos, pero también un campo de experimentación para distintos procedimientos didácticos. Por otra parte, Ana Lucía Frega percibió la necesidad de ampliar sus experiencias vitales y formativas, de manera que mientras se desempeñaba como docente de música, comenzó una búsqueda para adquirir nuevos conocimientos, cuestión que no era tan habitual en esos años. El hecho de compatibilizar la docencia en la escolaridad obligatoria con sus propios estudios de ampliación, probablemente influyó en buscar que las in-

\footnotetext{
${ }^{14}$ Abraham Jurafsky (1906-1993) fue compositor y director de orquesta. Fue también Director artístico de la Asociación Wagneriana de Buenos Aires. Como profesor participó en la formación musical de varias generaciones de músicos.

${ }^{15}$ La Escuela Argentina Modelo es un colegio privado de orientación católica, fundado en 1918 en Buenos Aires.
} 
terpretaciones musicales de sus alumnos, aunque fueran sencillas desde el punto de vista técnico, mantuvieran la calidad interpretativa.

Hay que tener en cuenta que en Argentina el ciclo lectivo iba, en las décadas de 1950 a 1990, desde marzo hasta diciembre. Así, después de trabajar como maestra de música durante varios años, comienza una formación complementaria internacional que constituye un elemento distintivo para las trayectorias vitales y profesionales la mayor parte de las profesoras de música de su época. Ana Lucía Frega, durante el período argentino de vacaciones estivales, viaja a Europa para conocer y estudiar con prestigiosas figuras: Maurice Martenot, Oliver Messiaen, Norbert Dufourq, Edgar Willems y Arnold Bentley, entre ellas.

Así, en febrero de 1964 conoció a Edgar Willems (1890-1978) en Ginebra, donde visitó tanto las clases de niños en la escuela de música, como el taller privado donde el maestro impartía diversas clases. Transcribimos las palabras de Ana Lucía Frega al respecto:

Tenía ahí su órgano con microtonos. Lo recuerdo siempre de pie y entregado. Conocimiento, convicción y entrega. Edgar Willems tenía un espacio para los niños pequeños (en grupos de diez), y tenía el armario con los objetos sonoros que empleaba en sus clases.

En París, en el Conservatorio Nacional de Música, señalados maestros dejan su huella y su ejemplo pedagógico: aunque como se ha mencionado no le era posible matricularse durante cursos completos, sino acudir como oyente a las clases durante del período estival argentino, de manera que durante las vacaciones escolares de verano ampliaba su formación y experiencias.

Transcribimos el testimonio de la Dra. Frega acerca de los maestros más destacados, todos ellos intérpretes, compositores, musicólogos, con un profundo dominio de la didáctica de la música:

De Oliver Messiaen, en primer lugar, destacaría su erudición. En segundo lugar, su intensidad. Había una gran convicción en el dictado de las clases. Recuerdo una clase sobre L'Escarbeau: el maestro miró el poema, la obra, y fueron a partir de ahí cuatro horas sin parar. Había mucho contenido, densidad 
en la clase. Explicaba, comentaba, tocaba el piano. Una gran entrega del maestro, era imposible aburrirse. En París yo vivía a dos cuadras de la Iglesia de la Trinidad, donde él era organista; él nos dijo el horario en el que iba a ensayar. Así que allí iba y lo escuchaba tocar el órgano. Yo todavía no era profesora en el Conservatorio Nacional, sino en la Escuela Argentina Modelo y en el Colegio Nacional de Quilmes. Hablo con vos y estoy viendo a Oliver Messiaen en el piano, siempre daba clase desde el piano.

Las clases del insigne organista y musicólogo Norbert Dufourq (19041990) también le provocaron una viva impresión:

Los sábados tenía clase de historia de la música con Norbert Dufourcq, que caminaba todo el tiempo, y era escuchar música, analizarla, y el profesor hablaba magistralmente. En su clase enorme había un órgano y Dufourc a veces tocaba para ejemplificar su explicación.

En esta evocación no puede faltar la figura de Maurice Martenot (1898-1980) quien ejerció una influencia notable en la perspectiva docente de la Ana Lucía Frega:

Maurice Martenot estaba marcado por la guerra y la resistencia. Le interesaba el orientalismo, el budismo, los vedas. En el transcurso de los dos meses y medio que ese año estuve en París realicé varias clases individuales, además de las del Conservatorio. Como maestro se interesaba por cada persona. Sus clases duraban cuatro horas (de 9 a 13); para Martenot era muy importante el valor del silencio.

Tanto Edgar Willems como Maurice Martenot contribuyeron a modelar la cosmovisión didáctica de Ana Lucía Frega, y, a través de su labor como formadora de docentes de música, en las siguientes generaciones de enseñantes. ${ }^{16}$

\footnotetext{
${ }^{16}$ Ana Lucía Frega, «A Comparison of the Teaching Strategies of Maurice Martenot and Edgar Willems: Conclusions and Implications for Future Research», Bulletin of the Council for Research in Music Education 127 (ISME Research Seminar. Winter, 1995/1996): 63-71.
} 


\section{DESCUBRIR EL CAMINO DE LA INVESTIGACIÓN}

Justamente, estos períodos de formación en Europa fueron los que iniciaron a Ana Lucía Frega en la metodología de investigación musical de forma sistemática, y los que llevaron, primero, a su presencia en los congresos mundiales de la International Society of Music Education (ISME), con un papel cada vez más importante, ${ }^{17}$ hasta llegar a ser electa Presidente en 1994.

En 1964 Ana Lucía Frega tuvo la oportunidad de viajar a la Universidad de Reading (Reino Unido) y conocer al Dr. Arnold Bentley (1913-2001):

Yo había leído y estudiado el libro con los papers del primer congreso de la ISME de 1953, y el Dr. Bentley me recomendó que fuera al Congreso que se iba a celebrar el año siguiente en Estados Unidos. En la Universidad de Reading, todas las semanas tenía una clase individual, de tipo tutoría. En su despacho, durante una hora y media, para comentar la bibliografía que me había dado para esa semana. Después le exponía las clases que había visto y comentábamos las cuestiones más importantes.

Este estudio sistemático de la investigación musical derivó en su asistencia al Congreso de la ISME de Estados Unidos en 1965, donde conoció a James Carlsen ${ }^{18}$ con quien tuvo una fructífera relación profesional en torno a la investigación musical, que la profesora Frega ha entendido siempre, según indicaba en $1998,{ }^{19}$ como una parte necesaria de la formación del músico:

En 1975 participé en el Seminario de Investigación en México, donde me dieron la palabra para que explicara el estado de la investigación musical en Argentina. Fue muy importante poder exponer todo lo que se estaba realizando. Después de esto viajé a Austria, donde conocí a la investigadora canadiense Margery Vaughan.

\footnotetext{
${ }^{17}$ https://www.isme.org/member/ana-lucía-frega.

${ }^{18}$ James Carlsen es profesor emérito de la Universidad de Washington; ha sido el editor del Journal of Research in Music Education.

${ }^{19}$ Ana Lucía Frega, «La investigación especializada en enseñanzas musicales», Musiker 10 (1998): 101-117.
} 
Esta mención a la Dra. Margery Vaughan resulta significativa, puesto que uno de los focos de interés de la Dra. Ana Lucía Frega ha sido la creatividad musical y su desarrollo, cuestión que se integra en sus publicaciones en especial a partir de ese momento. La investigadora canadiense Margery Vaughan se formó con Paul Torrance, y trabajó con Ana Lucía Frega tanto en labores de formación como de investigación en Canadá y en Argentina, de manera que influyó en investigadores y docentes de música argentinos a los que formó y asesoró, ${ }^{20}$ impulsando la integración de los procesos creativos en la docencia de música.

\section{INFLUENCIA DE LA EXPERIENCIA INTERNACIONAL EN SU PERSPECTIVA PROFESIONAL}

Ana Lucía Frega, a sus 82 años, es una de las figuras que ha tenido un papel influyente en el desarrollo de la didáctica de la música como disciplina durante varias décadas, no sólo en el ámbito de su propio país sino en un contexto internacional.

Son 61 años de trabajo. Tuve la posibilidad de ir directamente a las fuentes. Estuve frente a Martenot en sus clases. Estudié con una discípula de Justine Ward. Trabajé directamente con Madame Croptier. Con Willems. Eso da una perspectiva. Además, cada uno tenía sus propias vivencias. Martenot, que estaba marcado por la guerra y la resistencia. En clase éramos pocos, unas diez personas en clase con Messiaen. Los sábados tenía clase de historia de la música con Norbert Dufourcq, que caminaba todo el tiempo, y era escuchar música, analizarla, y el profesor hablaba magistralmente. Un salón en el viejo edificio del Conservatoire, en rue de Madrid 14 en Paris.

En su retrospectiva, Ana Lucía Frega valora especialmente la posibilidad de haberse nutrido directamente de las fuentes del conocimiento: el haber estudiado con personalidades musicales relevantes de su época. Desde nuestro punto de vista, el proceso interior tiene que dar un fruto. No solamente es importante haber podido trabajar directamente con

\footnotetext{
${ }^{20}$ La Dra. Margery Vaughan impartió en Buenos Aires cursos de formación sobre Creatividad Musical para docentes, entre 1983 y 1986, en la Escuela Argentina Modelo.
} 
tantas personas relevantes, sino también tener capacidad de interiorizar, y después aportar algo nuevo, construir a partir de esos aprendizajes y vivencias. Con respecto a esto, la Dra. Frega dice: « El alumno no tendría que reproducir el modelo. El profesor debe abrir ventanas y que cada uno elija sus caminos».

Para finalizar, subrayamos una idea que para la Dra. Ana Lucía Frega es muy importante, y que rescata de todos sus maestros y maestras, fundamental en el proceso de formación del músico: la exigencia de proporcionar el contexto necesario para desarrollar el pensamiento musical y explorar en el propio instrumento.

\section{CONCLUSIONES}

En este artículo, centrado en la primera etapa profesional de la Dra. Ana Lucía Frega (incluida su formación inicial, comienzos profesionales y entrada en la ISME), se ha dado una importancia fundamental al testimonio directo de la personalidad que es objeto de estudio. Sus años de formación corresponden a una época de florecimiento de los estudios de música entre la población de las cada vez más importantes clases medias argentinas, que buscaban una solidez tanto económica y como cultural.

En las palabras de Ana Lucía Frega se manifiesta una preocupación, desde muy joven, por la calidad de su docencia musical. Esta inquietud deviene en acción y se vuelca en el esfuerzo personal para formarse directamente con algunas de las máximas autoridades musicales del momento. Dentro de ese proceso de perfeccionamiento, se da cuenta de que la investigación es clave para afianzar y ampliar el conocimiento, y también busca una formación específica en este campo. El hecho de participar en la International Society of Music Education (ISME) en épocas en las que los profesores de música de habla hispana estaban escasamente representados internacionalmente, además de desempeñar papeles directivos en instituciones argentinas e internacionales, proporcionó a Ana Lucía Frega la oportunidad de influir en la formación de varias generaciones de docentes de música, en diversos niveles de la enseñanza.

$\mathrm{Al}$ recorrer la amplia producción bibliográfica de Ana Lucía Frega se pueden vislumbrar huellas de las enseñanzas de sus maestros, junto con 
el poso de su propia experiencia. Por esto, la insistencia en la práctica directa de la expresión musical, la audición atenta de obras musicales de alta calidad de diversos géneros, la reflexión personal a partir de las propias vivencias y del estudio sistemático de la música y de la bibliografía, y el ejercicio sistemático de la dimensión cognitiva, son conceptos recurrentes en sus textos.

Por otra parte, la Dra. Frega en sus propias reflexiones subraya la necesidad de que todo docente guíe para desarrollar la personalidad artística de alumnos y alumnas a partir de la audición reflexiva, la práctica musical y el estudio personal en un proceso de búsqueda de excelencia.

El punto de partida para esta trayectoria singular tiene que ver con el contacto con quienes habían generado metodologías clásicas de educación musical, que florecieron a lo largo del siglo xx. Un aspecto positivo de su personalidad es el interés por el estudio y la ampliación de sus conocimientos, así como su capacidad de interacción social, facilitada por su dominio de varias lenguas (francés, inglés, alemán).

Sin duda, hay más elementos que se entrelazan en la relevancia adquirida a lo largo de los años, que no serán objeto de este artículo, pero que sería interesante definir y considerar (contexto político argentino, desarrollo de líneas de trabajo en la ISME, relaciones personales, etc), con sus consecuentes controversias, luces y sombras, que serán ciertamente interesantes. Dejamos abierta la puerta a que otros investigadores continúen sobre estas cuestiones.

\section{Nota sobre la autora}

Susana Sarfson, nacida en Buenos Aires (Argentina), es Profesora Superior de Piano y de Clave (Medalla de oro), egresada del Conservatorio Nacional de Música "Carlos López Buchardo», así como Licenciada en Filología Hispánica, y Doctora en Filosofía y Ciencias de la Educación. En Argentina fue profesora del Collegium Musicum de Buenos Aires, Colegio Northlands, Conservatorio Nacional, Instituto Superior de Arte del Teatro Colón, entre otros. En España, es profesora titular de Didáctica de la Expresión Musical en la Universidad de Zaragoza (Facultad de Ciencias Humanas). Desarrolla tres líneas de investigación: historia de la educación musical, enseñanza de música para adultos mayores y recuperación 
de patrimonio musical barroco, que han originado diversas publicaciones (libros y artículos). Ha realizado estancias de investigación, docencia universitaria y participación en congresos en 19 países. Es miembro de la Sociedad Española de Musicología y de la International Society of Music Education. En sus años de formación, ha sido alumna de la Dra. Ana Lucía Frega, con quien también ha trabajado en el Instituto Superior de Arte del Teatro Colón, y en otros proyectos singulares a lo largo de muchas décadas, a quien agradece muy especialmente la realización de las entrevistas que originan este artículo.

\section{REFERENCIAS}

Bolaño Amigo, María Eugenia. «Estética ilustrada, discursos de clase y educación musical escolar en los comienzos del siglo xx». Historia y Memoria de la Educación 5 (2017): 385-400.

Cisterna CABRER, Francisco. «Categorización y triangulación como procesos de validación del conocimiento en investigación cualitativa». Theoria 14, no 1 (2005): 61-71.

Frega, Ana Lucía y Margery Vaughan. Creatividad musical. Fundamentos y estrategias para su desarrollo. Buenos Aires: Casa América, 1980.

Frega, Ana Lucía. "A Comparison of the Teaching Strategies of Maurice Martenot and Edgar Willems: Conclusions and Implications for Future Research». Bulletin of the Council for Research in Music Education 127 (ISME Research Seminar. Winter, 1995/1996): 63-71.

- Metodología comparada de la Educación Musical. Buenos Aires: Centro de Investigación Educativa Musical del Collegium Musicum, 1997.

- «La investigación especializada en enseñanzas musicales». Musiker 10 (1998): 101-117.

- Didáctica de la Música: Las enseñanzas musicales en perspectiva. Buenos Aires: Bonum, 2007.

- «Creatividad en enseñanzas artísticas». Journal for Educators, Teachers and Trainers 5, no. 1 (2013): 6-8.

Gallo, Ezequiel. La Pampa gringa: La colonización agrícola en Santa Fe (18701895). Buenos Aires: Instituto Torcuato Di Tella, 1984.

GARCía CÁNEPA, J. «La enseñanza musical en Argentina: el Conservatorio Nacional de Música Carlos López Buchardo». Conservatorianos 6 (2000): 53-57.

Martínez Miguélez, Miguel. El método etnográfico de investigación. 2005. http://investigacionypostgrado.uneg.edu.ve/intranetcgip/documentos/225000/225000archivo00002.pdf. (Consultado el 23-07- 2017) 
Ruíz Olabuénaga, José Ignacio. «Historias de vida». En Metodología de la Investigación Cualitativa, editado por José I. Ruíz Olabuénaga, 207-306. Bilbao: Universidad de Deusto, 2012.

SAítTA, Sylvia. Crítica en la constitución del periodismo moderno. Buenos Aires: Universidad de Buenos Aires, 1996.

SARFSON GLEIZER, Susana. Educación Musical en Aragón (1900-1950). Legislación, publicaciones y escuela. Zaragoza: Prensas Universitarias. 2010.

Vaughan, Margery. «Music for Gifted children: A bridge to consciousness». Music Educators Journal 58 (1972): 70-74.

VERAs, Eliane. «Historia de vida: ¿un método para las ciencias sociales?». Cinta Moebio 39 (2010): 142-152.

ZDZINSKI, Stephen. «Joseph A. Labuta and His Life in Music Education: An Oral History». Journal of Historical Research in Music Education XxIx, no. 2 (2008): 108-128. 